\title{
Optimisation of Effective Design Parameters for an Automotive Transmission Gearbox to Reduce Tooth Bending Stress
}

\author{
Mehmet Bozca \\ Machine Design Division, Mechanical Engineering Faculty, Yildiz Technical University, Istanbul, Turkey \\ Email: mbozca@yildiz.edu.tr
}

How to cite this paper: Bozca, M. (2017) Optimisation of Effective Design Parameters for an Automotive Transmission Gearbox to Reduce Tooth Bending Stress. Modern Mechanical Engineering, 7, 35-56. https://doi.org/10.4236/mme.2017.72004

Received: February 15, 2017

Accepted: May 7, 2017

Published: May 10, 2017

Copyright $\odot 2017$ by author and Scientific Research Publishing Inc. This work is licensed under the Creative Commons Attribution International License (CC BY 4.0).

http://creativecommons.org/licenses/by/4.0/ (c) (i) Open Access

\begin{abstract}
Optimisation of effective design parameters to reduce tooth bending stress for an automotive transmission gearbox is presented. A systematic investigation of effective design parameters for optimum design of a five-speed gearbox is studied. For this aim contact ratio effect on tooth bending stress by the changing of contact ratio with respect to pressure angle is analysed. Additionally, profile modification effects on tooth bending stress are presented. During the optimisation, the tooth bending stress is considered as the objective function, and all the geometric design parameters such as module, teeth number etc. are optimised under two different constraints, including tooth contact stress and constant gear centre distance. It can be concluded that higher the contact ratio results in a reduced tooth bending stress, while higher the pressure angle caused an increase in tooth bending stress and contact stress, since decreases in the contact ratio. In addition, application of positive profile modification on tooth reduces tooth bending stress. All of the obtained optimum solutions satisfy all constraints.
\end{abstract}

\section{Keywords}

Optimisation, Gears, Pressure Angle, Contact Ratio, Bending Stress, Contact Stress

\section{Introduction}

The purpose of this study is optimisation of effective design parameters to reduce tooth bending stress for an automotive transmission gearbox.

Gears are mechanically transmitted power in automotive transmissions. Therefore, determining the geometric design parameters of gears is crucial.

By optimising all the geometric parameters of the gears, obtaining desired 
gearbox structures can be possible.

All constraints are also satisfied by the optimised geometric design parameters, based on pressure angle.

By optimising the effective geometric design parameters of the five-speed gearbox, such as the module, number of teeth, etc., reducing the tooth bending stress is possible.

Increasing the contact ratio results in reduced tooth bending stress and tooth contact stress. However, increased the pressure angle causes increasing of the tooth bending stress and tooth contact stress, since the contact ratio reduces depending on increasing of the pressure angle. Furthermore, higher contact ratio has a positive effect on reducing tooth bending stress. In contrast, higher pressure angle has a negative effect on reducing tooth bending stress. Application of tooth profile modification has a positive effectiveness on reducing the tooth bending stress.

The following discussion summarises findings from the literature:

\subsection{Literature Review}

The following results on tooth bending strength are presented in the literature:

An asymmetric gear pair improves the tooth-root bending load carrying capacity of the pinion and wheel gear at higher pressure angles on the coast side compared to a conventional symmetric gear. The optimum profile shift values increases with an increase in the speed ratio and number of teeth in the pinion, and increasing the asymmetric factor and pressure angles on the drive side improves the tooth-root bending capacity. When the speed ratio increases, the optimum maximum fillet stress increases very slightly compared to that of optimum profile shift factor for pinion [1].

Asymmetric involute-type teeth were studied, since the non-involute teeth application has a number of disadvantages. The concept of one-sided involute asymmetric spur gear teeth is to increase the load carrying capacity of the driving involute. The literature concludes that the load carrying capacity can increase to $28 \%$ higher than that of standard $20^{\circ}$ involute teeth [2].

The advantage of using proposed asymmetric design in gearboxes is increased bending strength, pitting resistance, without changing the dimension or number of teeth in the gearbox [2].

An alternative method to increase the tooth bending strength of involute gear teeth is positive modification of addendum (positive shifting) the pinion and, in some cases, mating wheel. This method produces well-running teeth, but both the pitting resistance and scoring resistance are reduced due to the positive shifting [2].

A smaller pressure angle causes to produce undercut for a given number of teeth. However, the contact ratio increases, and load carrying capacity may be improved [3].

Tooth profile modification is an effective parameter for optimising the geometric design parameters of gears. A numerical study found that the application 
of positive profile modification results in reduced tooth bending stress and increased safety factor for tooth bending stress [4].

\subsection{Gearbox Mechanism}

The gearbox mechanism is shown in Figure 1. Where $Z_{1 p}, Z_{2 p}, Z_{3 p}$ and $Z_{4 p}$ denotes the $1^{\text {st }}$ speed pinion gear, the $2^{\text {nd }}$ speed pinion gear, the $3^{\text {rd }}$ speed pinion gear and the $4^{\text {th }}$ speed pinion gear respectively. $Z_{C p}$ and $Z_{R p}$ denote the constant speed pinion gear and the rear speed pinion gear. $Z_{\mathrm{g} 1}, Z_{\mathrm{g} 2}, Z_{\mathrm{g} 3}$ and $Z_{\mathrm{g} 4}$ denotes the $1^{\text {st }}$ speed wheel gear, the $2^{\text {nd }}$ speed wheel gear, the $3^{\text {rd }}$ speed wheel gear and the $4^{\text {th }}$ speed wheel gear respectively. $Z_{\mathrm{Cg}}$ and $Z_{\mathrm{Rg}}$ denote the constant speed wheel gear and the rear speed wheel gear. $S_{1}, S_{2}$ and $S_{3}$ denote synchronisers.

\section{Effective Geometric Design Parameters}

General definitions and specification factors for gears are given in DIN 868 as follows.

The module, $m$ is the basic parameter for the linear dimensions of gear tooth systems. It is the result of dividing the pitch, $p$ by the number $\pi$. The pitch is determined by the dimensions of the datum surface and the number of teeth; see Figure 2(a).

The number of teeth, $z$ of a gear is the number of teeth present on the full circumference of the gear or the number that would be feasible for a chosen pitch; see Figure 2(b).

The face width, $b$, is the distance between the two end surfaces of the gear tooth system; see Figure 2(c).

The helix angle, $\beta$, is the angle between the helix line and horizontal axis; see Figure 2(d).

The centre distance, $a$, of a gear pair with parallel axes is the shortest distance between the two axes; see Figure 2(e).

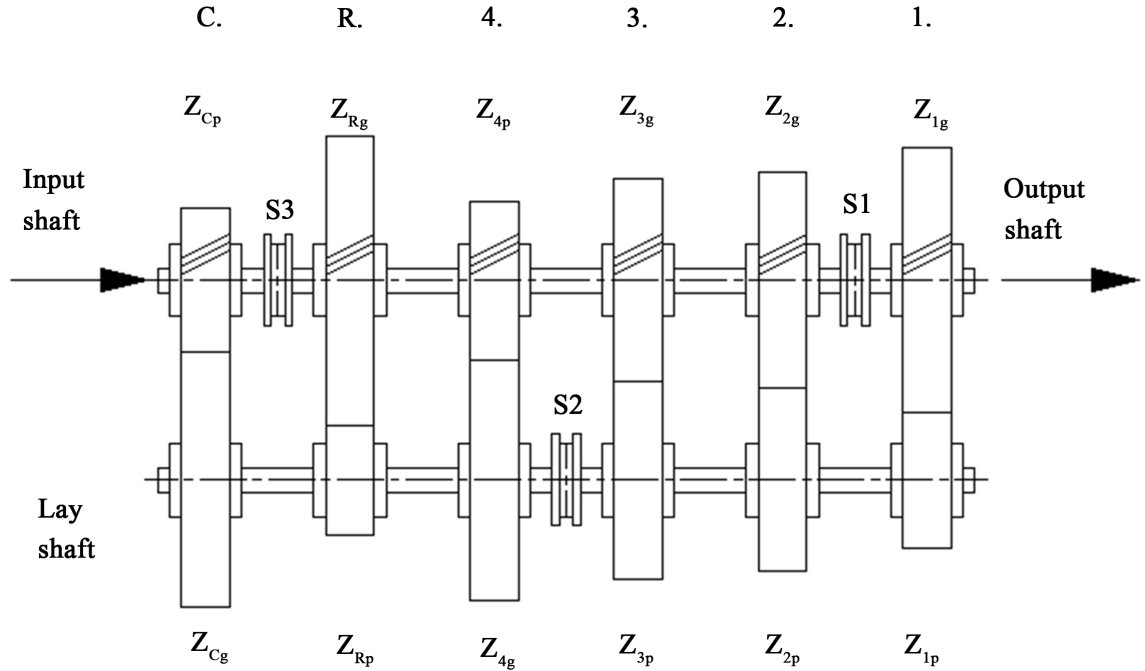

Figure 1. Five-Speed manual gearbox with helical gear for automotive transmission. 


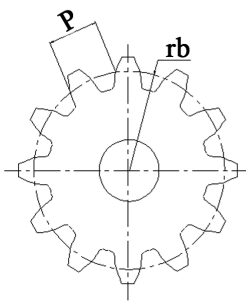

(a)

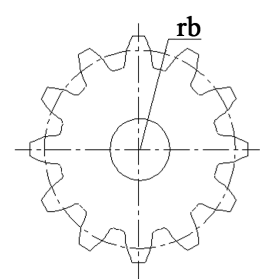

(b)

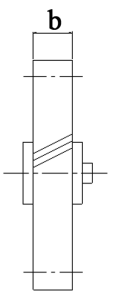

(c)

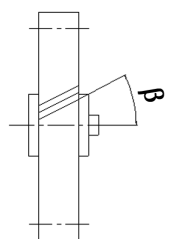

(d)

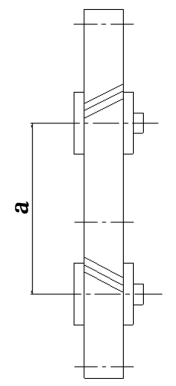

(e)

Figure 2. Design parameters for a gearbox. (a) module; (b) number of teeth $z$; (c) facewidth b; (d) helix angle $\beta$; (e) centre distance a.

\section{Contact Ratio}

The dimensions of helical gear are shown in Figure 3 and the contact line is shown Figure 4. Obviously, tooth profiles must be proportioned such that a second pair of mating teeth comes into contact before the first pair is out of contact [5].

If the gear contact ratio equal to 1 , then one tooth is leaving contact just as the next is beginning contact. A unity contact angle is undesirable, because slight errors in tooth spacing will cause oscillations in velocity, and, subsequently, vibration, and noise. In addition, the load will be applied on the tip of the tooth, creating the largest possible bending moment [6].

In general, the higher the contact ratio, the smoother the running of the gears. When a contact ratio is equal to 2 or more means that at least two pairs of teeth are theoretically in contact currently [5].

If a profile contact ratio is lower than 2.0, is called as Low Contact Ratio (LCR), while gearing with this parameter equal to 2.0 or greater than 2.0 is called as High Contact Ratio (HCR) [5].

The contact ratio consists of two parts, such as the transverse contact ratio, $\varepsilon_{\alpha}$, and the overlap (face contact) ratio, $\varepsilon_{\beta}$.

\subsection{Transverse Contact Ratio, $\varepsilon_{\alpha}$}

The contact ratio (CR) is defined as the average number of teeth in contact during the gear rotation. The transverse contact ratio, $\varepsilon_{\alpha}$ is calculated as follows [8].

$$
\varepsilon_{\alpha}=\frac{g_{\alpha}}{p_{e t}}=\frac{0.5 \cdot\left(\sqrt{d_{a 1}^{2}-d_{b 1}^{2}}+\sqrt{d_{a 2}^{2}-d_{b 2}^{2}}\right)-a_{d} \div \sin \alpha_{t}}{\pi \cdot m_{t} \cdot \cos \alpha_{t}}
$$

where $g_{\alpha}$ is the path length of the contact line [mm], and $p_{e t}$ is the base pitch $[\mathrm{mm}], d_{a 1}$ is the addendum circle diameter of the pinion gear $[\mathrm{mm}], d_{b 1}$ is the base circle diameter of the pinion gear $[\mathrm{mm}], d_{a 2}$ is the addendum circle diameter of the wheel gear $[\mathrm{mm}], d_{b 2}$ is the base circle diameter of the wheel gear $[\mathrm{mm}], a_{d}$ is the centre distance $[\mathrm{mm}], \alpha_{t}$ is the transverse pressure angle $\left[{ }^{\circ}\right]$, and $m_{t}$ is the transverse module $[\mathrm{mm}]$.

The addendum circle diameter of the pinion gear, $d_{a 1}$, is calculated as follows [8]. 

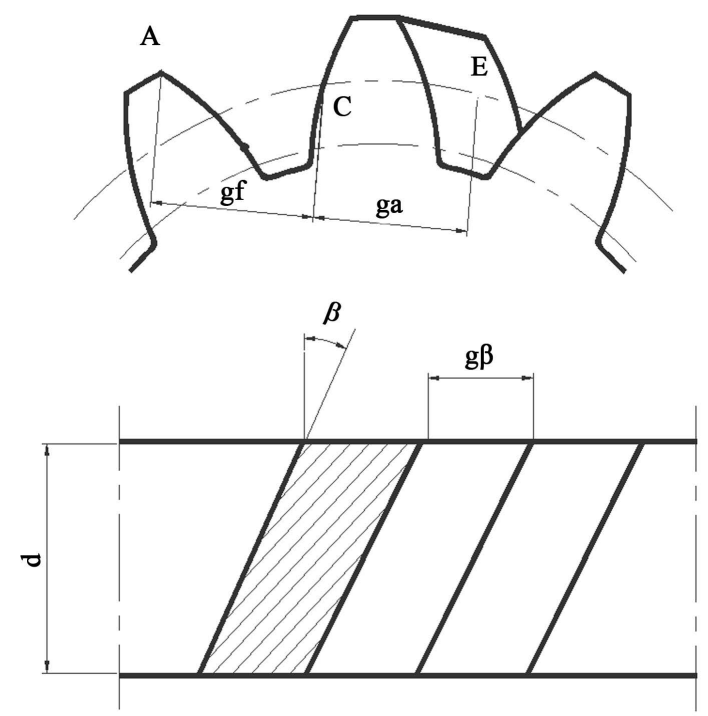

Figure 3. Contac line of helical gear.

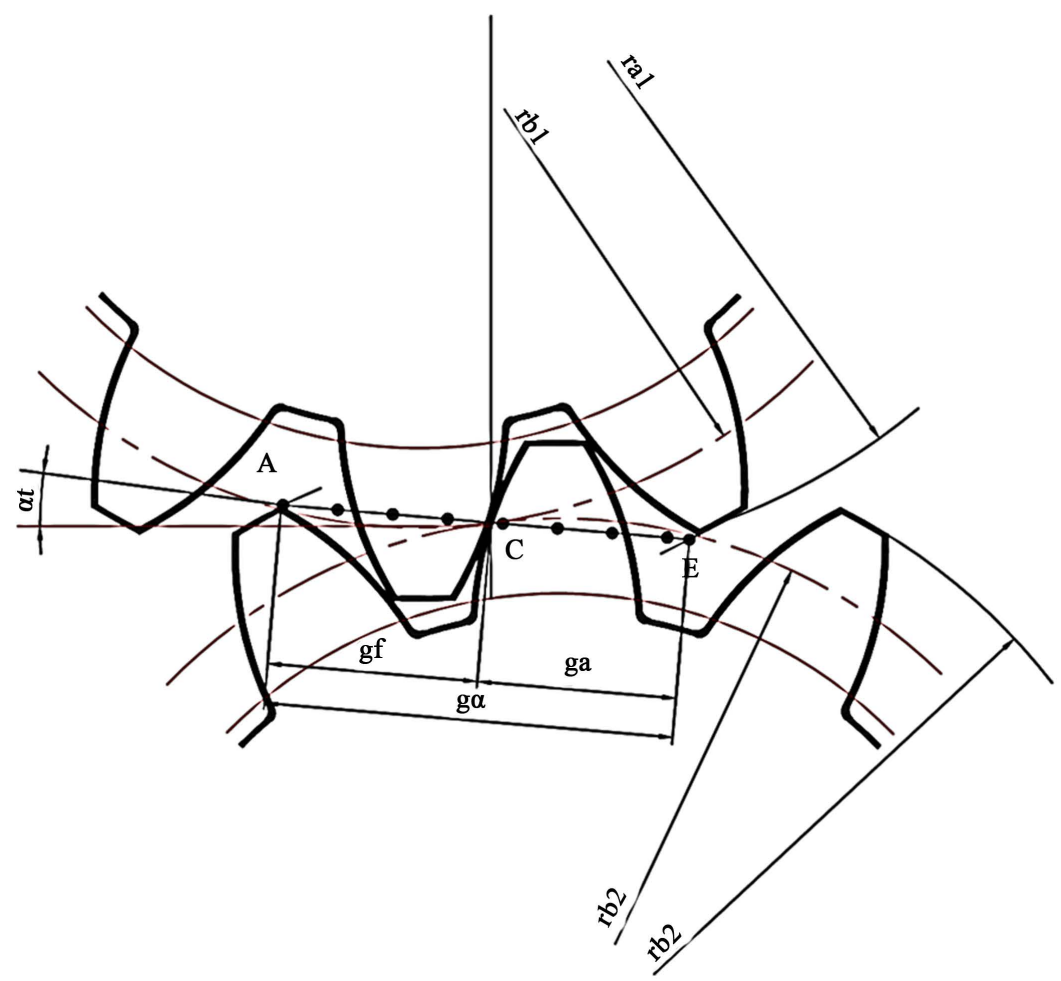

Figure 4. Contac line of helical gear including contact length AE.

$$
d_{a 1}=d_{1}+2 \cdot h_{a}=d_{1}+2 \cdot m_{n}=m_{n} \cdot\left(2+\frac{z}{\cos \beta}\right)
$$

where $m_{n}$ is the normal module [mm], $z$ is the number teeth [-], and $\beta$ is the helix angle $\left[{ }^{\circ}\right]$.

The base circle diameter of the pinion gear, $d_{b 1}$, is calculated as follows [8].

$$
d_{b 1}=d_{1} \cdot \cos \alpha_{t}=z \cdot \frac{m_{n}}{\cos \beta} \cdot \cos \alpha_{t}
$$


The addendum circle diameter of the wheel gear, $d_{a 2}$, is calculated as follows $[8]$.

$$
d_{a 2}=d_{2}+2 \cdot h_{a}=d_{2}+2 \cdot m_{n}=m_{n} \cdot\left(2+\frac{z}{\cos \beta}\right)
$$

The base circle diameter of the wheel gear, $d_{b 2}$, is calculated as follows [9].

$$
d_{b 2}=d_{2} \cdot \cos \alpha_{t}=z \cdot \frac{m_{n}}{\cos \beta} \cdot \cos \alpha_{t}
$$

The centre distance, $a_{d}$ is calculated as follows [8].

$$
a_{d}=\frac{d_{1}+d_{2}}{2}=m_{t} \cdot \frac{\left(z_{1}+z_{2}\right)}{2}=\frac{m_{n}}{\cos \beta} \frac{\left(z_{1}+z_{2}\right)}{2}
$$

\subsection{Overlap Ratio, $\varepsilon_{\beta}$}

The overlap ratio, $\varepsilon_{\beta}$ is calculated as follows [8].

$$
\varepsilon_{\beta}=\frac{U}{p_{t}}=\frac{b \cdot \tan \beta}{p_{t}}=\frac{b \cdot \sin \beta}{\pi \cdot m_{n}}
$$

where $U$ is the action length $[\mathrm{mm}], p_{t}$ is the transverse pitch $[\mathrm{mm}], b$ is the face width [mm], and $m_{n}$ is the normal module [mm].

\subsection{Total Contact Ratio, $\varepsilon_{\gamma}$}

The total contact ratio, $\varepsilon_{\gamma}$ is calculated as follows.

$$
\varepsilon_{\gamma}=\varepsilon_{\alpha}+\varepsilon_{\beta}
$$

where $\varepsilon_{\alpha}$ is the transverse contact ratio and $\varepsilon_{\beta}$ is the overlap ratio. Helical gears have higher contact ratio than spur gears thus, they have also higher load carrying capacities than spur gears.

\section{Strength of Helical Gears}

The gear strength is defined by two criteria such as the tooth bending strength and tooth contact strengths according to the ISO 6336.

\subsection{Tooth Bending Stress}

The bending stress in distribution are shown in Figure 5. The real tooth-root stress, $\sigma_{F}$ is calculated as follows [7] [8]

$$
\sigma_{F}=\frac{F_{t}}{b m_{n}} Y_{F} Y_{S} Y_{\varepsilon} Y_{\beta} K_{A} K_{V} K_{F \beta} K_{F \alpha}
$$

The permissible bending stress, $\sigma_{F p}$, is calculated as follows [7] [8].

$$
\sigma_{F p}=\sigma_{F \lim } Y_{S T} Y_{N} Y_{\delta} Y_{R} Y_{X}
$$

where all the responsible parameters for the tooth bending stress are given in Table 1. The safety factor for bending stress $S_{F}$ is calculated as follows [7] [8]

$$
S_{F}=\frac{\sigma_{F p}}{\sigma_{F}}
$$


Table 1. Tooth bending stress parameters.

\begin{tabular}{|c|c|c|c|c|c|c|}
\hline Parameters & $1^{\text {st }}$ pinion & $2^{\text {nd }}$ pinion & $3^{\text {rd }}$ pinion & $4^{\text {th }}$ pinion & Constant pinion & Rear pinion \\
\hline Torque $\mathrm{T}_{\mathrm{L}}[\mathrm{N} . \mathrm{mm}]$ & $392 \times 10^{3}$ & $392 \times 10^{3}$ & $316 \times 10^{3}$ & $252 \times 10^{3}$ & $200 \times 10^{3}$ & $900 \times 10^{3}$ \\
\hline gear ratio $\mathrm{u}$ & 1.814 & 1.147 & 1.242 & 1.560 & 1 & 2.84 \\
\hline stress correction factor $\mathrm{Y}_{\mathrm{ST}}$ & 2 & 2 & 2 & 2 & 2 & 2 \\
\hline form factor $Y_{F}$ & 2.75 & 2.75 & 2.75 & 2.75 & 2.75 & 2.75 \\
\hline stress correction factor $Y_{S}$ & 1.60 & 1.60 & 1.60 & 1.60 & 1.60 & 1.60 \\
\hline application factor $\mathrm{K}_{\mathrm{A}}$ & 1.25 & 1.25 & 1.25 & 1.25 & 1.25 & 1.25 \\
\hline internal dynamic factor $K_{V}$ & 1.14 & 1.14 & 1.14 & 1.14 & 1.14 & 1.14 \\
\hline transverse load factor for tooth-root stress $\mathrm{K}_{\mathrm{F} a}$ & 1.2 & 1.2 & 1.2 & 1.2 & 1.2 & 1.2 \\
\hline permissible bending stress $\sigma_{\mathrm{FLim}}\left[\mathrm{N} / \mathrm{mm}^{2}\right]$ & 500 & 500 & 500 & 500 & 500 & 500 \\
\hline life factor for tooth-root stress $\mathrm{Y}_{\mathrm{N}}$ & 1 & 1 & 1 & 1 & 1 & 1 \\
\hline relative notch sensitivity factor $Y_{\delta}$ & 1 & 1 & 1 & 1 & 1 & 1 \\
\hline relative surface factor $Y_{R}$ & 1 & 1 & 1 & 1 & 1 & 1 \\
\hline size factor relevant to tooth-root strength $Y_{X}$ & 1 & 1 & 1 & 1 & 1 & 1 \\
\hline
\end{tabular}

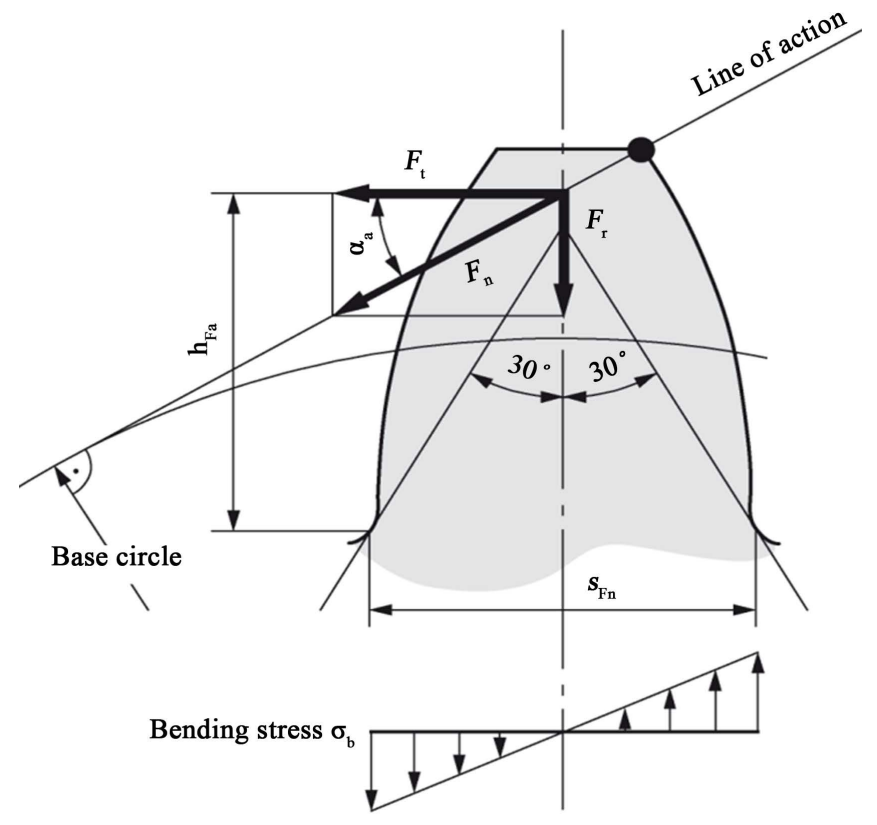

Figure 5. Bending stress at the tooth root.

\subsection{Tooth Contact Stress}

The contact stress, distribution is shown in Figure 6. The real contact stress, $\sigma_{H}$ is calculated as follows [7] [8]

$$
\sigma_{H}=\sqrt{\frac{F_{t}}{b m_{n}} \frac{u+1}{u}} Z_{H} Z_{E} Z_{\varepsilon} Z_{\beta} \sqrt{K_{A} K_{V} K_{H \beta} K_{H \alpha}}
$$

The permissible contact stress, $\sigma_{H p}$ is calculated as follows [7] [8]:

$$
\sigma_{H p}=\sigma_{H \lim } Z_{N} Z_{L} Z_{V} Z_{R} Z_{W} Z_{X}
$$

where all the responsible parameters for the tooth contact stress are given in Table 2 . 
Table 2. Tooth contact stress parameters.

\begin{tabular}{|c|c|c|c|c|c|c|}
\hline Parameters & $1^{\text {st }}$ pinion & $2^{\text {nd }}$ pinion & $3^{\text {rd }}$ pinion & $4^{\text {th }}$ pinion & Constant pinion & Rear pinion \\
\hline Torque $\mathrm{T}_{\mathrm{L}}[\mathrm{N} . \mathrm{mm}]$ & $392 \times 10^{3}$ & $392 \times 10^{3}$ & $316 \times 10^{3}$ & $252 \times 10^{3}$ & $200 \times 10^{3}$ & $900 \times 10^{3}$ \\
\hline gear ratio u & 1.814 & 1.147 & 1.242 & 1.560 & 1 & 2.84 \\
\hline zone factor $Z_{H}$ & 1 & 1 & 1 & 1 & 1 & 1 \\
\hline elasticity factor $Z_{E}$ & 189.8 & 189.8 & 189.8 & 189.8 & 189.8 & 189.8 \\
\hline transverse load factor for contact stress $K_{H \alpha}$ & 1.2 & 1.2 & 1.2 & 1.2 & 1.2 & 1.2 \\
\hline permissible contact stress $\sigma_{\text {Hlim }}\left[\mathrm{N} / \mathrm{mm}^{2}\right]$ & 1400 & 1400 & 1400 & 1400 & 1400 & 1400 \\
\hline life factor for contact stress $Z_{N}$ & 1 & 1 & 1 & 1 & 1 & 1 \\
\hline velocity factor $Z_{V}$ & 1 & 1 & 1 & 1 & 1 & 1 \\
\hline roughness factor $Z_{R}$ & 1 & 1 & 1 & 1 & 1 & 1 \\
\hline work hardening factor $\mathrm{Z}_{\mathrm{W}}$ & 1 & 1 & 1 & 1 & 1 & 1 \\
\hline size factor for contact stress $Z_{X}$ & 1 & 1 & 1 & 1 & 1 & 1 \\
\hline
\end{tabular}

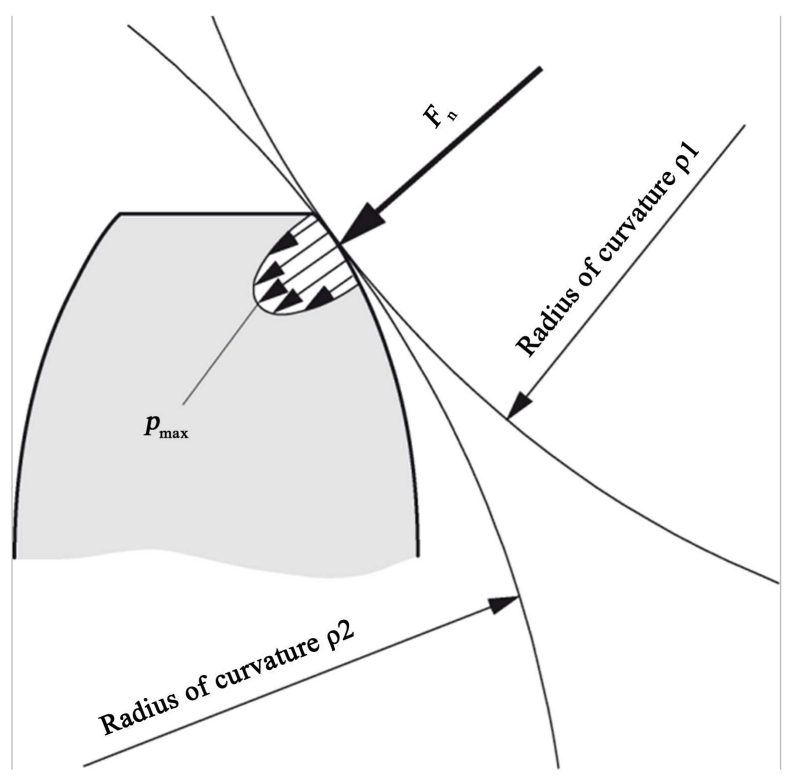

Figure 6. Contact stress at the tooth flank.

The safety factor for contact stress, $S_{H}$, is calculated as follows [7] [8]:

$$
S_{H}=\frac{\sigma_{H p}}{\sigma_{H}}
$$

\section{Optimisation of Effective Design Parameters of Gearbox}

Constrained optimisation method is helpful for designing light-weight gearbox structures. Constraints, including tooth contact stress and constant distance between gear centres can be used for this optimisation.

During optimisation, the aim is typically to minimise the cost of a structure while satisfying all the design requirements. By optimising the effective design 
parameters, a light-weight gearbox structure design is also possible [9] [10].

\subsection{Objectives Function}

Tooth bending stresses are considered as objective functions, during the optimisation study. The flowchart of the optimisation procedure of geometric design parameters is shown in Figure 7. The following objective function was used:

$$
F=\min (\sigma)
$$

Following minimum tooth bending stress is defined as objective function:

$$
\min \left(\sigma_{F}\right)=\min \left(\frac{F_{t}}{b m_{n}} Y_{F} Y_{S} Y_{\varepsilon} Y_{\beta} K_{A} K_{V} K_{F \beta} K_{F \alpha}\right)
$$

Thus, the module $m$, the number of teeth $z$, and the helix angle $\beta$, are the design parameters to be determined. During the constrained optimisation, the following optimisation problem is solved:

$$
\min \sigma(m, z, \beta, b)
$$

Subject to: $\mathbf{L B} \leq m, z, \beta, b \leq \mathbf{U B}$ and $G(X) \leq 0$

where LB is lower bound and UB is upper bounds on the design parameter vector. The iterations start with the initial values of design parameters such as, $m_{0}$, $z_{0}, \beta_{0}$, and $b_{0}$. Initial design parameters $\mathrm{X} 0$ are varied during the optimisation process, where $\mathrm{G}(\mathrm{X}) \leq 0$ is the nonlinear inequalities.

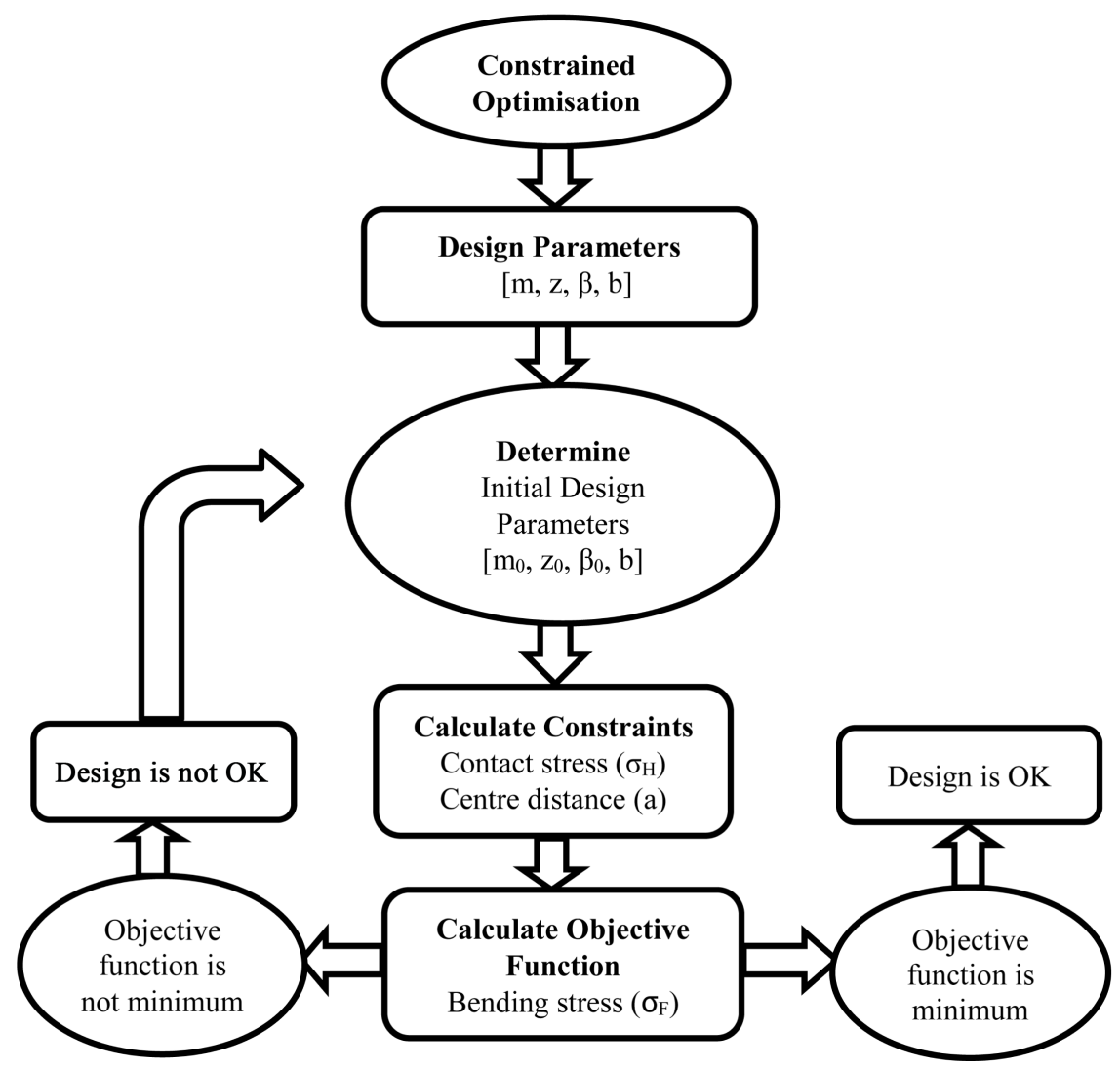

Figure 7. Flow chart to optimise gearbox design parameters. 


\subsection{Constraint Functions}

During constraint optimisation, the tooth contact stress and constant distance between gear centres are considered as the constraint function as follows:

$$
\sigma_{H}-\sigma_{H p} \leq 0
$$

where $\sigma_{H}$ is the real contact stress $\left[\mathrm{N} / \mathrm{mm}^{2}\right]$ and $\sigma_{H p}$ is the permissible contact stress $\left[\mathrm{N} / \mathrm{mm}^{2}\right]$.

$$
a_{1}=a_{2}=a_{3}=a_{4}=a_{5}=a_{R}=\text { constant }
$$

where $a_{1}$ is the centre distance of the $1^{\text {st }}$ speed, $a_{2}$ is the centre distance of the $2^{\text {nd }}$ speed, $a_{3}$ is the centre distance of the $3^{\text {rd }}$ speed, $a_{4}$ is the centre distance of the $4^{\text {th }}$ speed, $a_{5}$ is the centre distance of the $5^{\text {th }}$ speed and $a_{R}$ is the centre distance of the rear speed.

\section{Numerical Example}

Constrained optimisation method is applied to the five-speed gearbox mechanism to reduce tooth bending stress. All optimisation programs are developed using MATLAB. The sequential quadratic programming (SQP) method is used.

Twenty-four design parameters are optimised simultaneously using the developed programs. All the parameters for the tooth strength calculation are shown in Table 1 and Table 2, respectively.

\section{Results}

It is observed in solution 1 (Table 3 ) that the obtained optimum effective parameters result in satisfied values for each speed. By considering safety factors, this solution is more acceptable for $1^{\text {st }}$ and rear speed. The safety factor for bending stress, $S_{F}$, ranges between 1.1797 and 3.1783 , and the safety factor for contact stress, $\mathrm{S}_{\mathrm{H}}$, varies between 1.2269 and 2.5490 .

It is observed in solution 2 (Table 3 ) that the obtained optimum effective parameters result in acceptable values for each speed. The safety factor for bending stress, $\mathrm{S}_{\mathrm{F}}$, ranges between 1.1254 and 3.0457, and the safety factor for contact stress, $\mathrm{S}_{\mathrm{H}}$, varies between 1.1854 and 2.4725 .

The results from solution 3 (Table 3 ) show that the obtained optimum effective parameters satisfy desired requirements. By considering safety factors, this solution is more acceptable for $2^{\text {nd }}$ and $3^{\text {rd }}$ speed. The safety factor for bending stress, $S_{F}$, ranges between 1.0776 and 2.9275, and the safety factor for contact stress, $\mathrm{S}_{\mathrm{H}}$, varies between 1.1491 and 2.4046.

The results from solution 4 (Table 3 ) indicate that the obtained optimum effective parameters satisfy all requirements. The safety factor for bending stress, $\mathrm{S}_{\mathrm{F}}$, ranges between 1.0357 and 2.8229 , and the safety factor for contact stress, $\mathrm{S}_{\mathrm{H}}$, varies between 1.1175 and 2.3448 .

The results from solution 5 (Table 3 ) show that the obtained optimum solutions result in desired values. However, by considering safety factors, this solution is more acceptable for constant pinion. The safety factor for bending stress, $\mathrm{S}_{\mathrm{F}}$, ranges between 0.9993 and 2.7314 , and the safety factor for contact stress, $\mathrm{S}_{\mathrm{H}}$, varies between 1.0901 and 2.2926. 
Table 3. (a) Optimisation results-Solution no 1; (b) Optimisation results-Solution no 2; (a) Optimisation results-Solution no 3; (d) Optimisation results-Solution no 4; (e) Optimisation results-Solution no 5; (f) Optimisation results-Solution no 6.

(a)

Solution no 1 (pressure angle $\alpha=12^{\circ}$ )

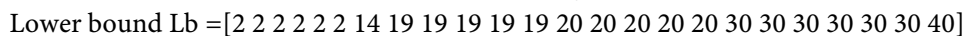

Upper bound Ub = [7 77777141919191919323232323434343434343442$]$

Initial condition X0 = [7 777771419191919193131313131323333323232 42]

\begin{tabular}{|c|c|c|c|c|c|c|}
\hline & $1^{\text {st }}$ pinion & $2^{\text {nd }}$ pinion & $3^{\text {rd }}$ pinion & $4^{\text {th }}$ pinion & Constant pinion & Rear pinion \\
\hline Module m & 4.4442 & 3.3709 & 3.2283 & 2.8281 & 3.6180 & 2.7141 \\
\hline Number of teeth $\mathrm{z}$ & 14.000 & 19.000 & 19.000 & 19.000 & 19.000 & 19.000 \\
\hline Helix angle $\beta$ & 32.000 & 30.7483 & 30.7391 & 30.7133 & 30.7645 & 31.6943 \\
\hline Face width $b$ & 34.000 & 33.000 & 32.000 & 32.000 & 32.000 & 44.000 \\
\hline Pressure angle $\alpha_{t}$ & 14.0709 & 13.8919 & 13.8906 & 13.8871 & 13.8942 & 14.0261 \\
\hline Centre distance a & 80.000 & 80.000 & 80.000 & 80.000 & 80.000 & 80.000 \\
\hline Transverse contact ratio $\varepsilon_{\alpha}$ & 1.7836 & 1.8255 & 1.8423 & 1.8895 & 1.7963 & 1.8913 \\
\hline Overlap ratio $\varepsilon_{\beta}$ & 1.6651 & & & & & \\
\hline Bending stress $\sigma_{\mathrm{F}}$ & 822.2394 & 679.0009 & 692.3186 & 558.4413 & 314.6310 & 847.6631 \\
\hline Safety factor for bending stress $S_{F}$ & 1.2162 & 1.4728 & 1.4444 & 1.7907 & 3.1783 & 1.1797 \\
\hline Contact stress $\sigma_{\mathrm{H}}$ & 921.600 & 780.1000 & 726.1000 & 697.2000 & 549.2000 & 1141.100 \\
\hline Safety factor for contact stress $\sigma_{\mathrm{H}}$ & 1.5191 & 1.7946 & 1.9280 & 2.0081 & 2.5490 & 1.2269 \\
\hline
\end{tabular}

(b)

Solution no 2 (pressure angle $\alpha=14^{\circ}$ )

Lower bound $\mathrm{Lb}=$ [as same as above]

Upper bound $\mathrm{Ub}=$ [as same as above]

Initial condition $\mathrm{X} 0=[$ as same as above $]$

\begin{tabular}{|c|c|c|c|c|c|c|}
\hline & $1^{\text {st }}$ pinion & $2^{\text {nd }}$ pinion & $3^{\text {rd }}$ pinion & $4^{\text {th }}$ pinion & Constant pinion & Rear pinion \\
\hline Module m & 3.4442 & 3.3708 & 3.2282 & 2.8280 & 3.6179 & 2.7140 \\
\hline Number of teeth $\mathrm{z}$ & 14.000 & 19.000 & 19.000 & 19.000 & 19.000 & 19.000 \\
\hline Helix angle $\beta$ & 32.000 & 30.7513 & 30.7423 & 30.7172 & 30.7671 & 31.6986 \\
\hline Face width $b$ & 34.000 & 33.000 & 32.000 & 32.000 & 32.000 & 44.0000 \\
\hline Pressure angle $\alpha_{t}$ & 16.3835 & 16.1785 & 16.1771 & 16.1731 & 16.1810 & 16.3329 \\
\hline Centre distance a & 80.000 & 80.000 & 80.000 & 80.000 & 80.000 & 80.000 \\
\hline Transverse contact ratio $\varepsilon_{\alpha}$ & 1.6768 & 1.7140 & 1.7277 & 1.7656 & 1.6901 & 1.7655 \\
\hline Overlap ratio $\varepsilon_{\beta}$ & 1.6651 & & & & & \\
\hline Bending stress $\sigma_{\mathrm{F}}$ & 858.9134 & 709.4125 & 723.8493 & 585.0816 & 328.3350 & 888.5450 \\
\hline Safety factor for bending stress $S_{F}$ & 1.1643 & 1.4096 & 1.3815 & 1.7092 & 3.0457 & 1.1254 \\
\hline Contact stress $\sigma_{\mathrm{H}}$ & 950.500 & 805.100 & 749.800 & 721.200 & 566.200 & 1181.000 \\
\hline Safety factor for contact stress $\sigma_{\mathrm{H}}$ & 1.4729 & 1.7389 & 1.8671 & 1.9412 & 2.4725 & 1.1854 \\
\hline
\end{tabular}


(c)

\begin{tabular}{|c|c|c|c|c|c|c|}
\hline \multicolumn{7}{|c|}{$\begin{array}{c}\text { Solution no } 3 \text { (pressure angle } \alpha=16^{\circ} \text { ) } \\
\text { Lower bound } \mathrm{Lb}=[\text { as same as above }] \\
\text { Upper bound } \mathrm{Ub}=[\text { as same as above }] \\
\text { Initial condition } \mathrm{X} 0=[\text { as same as above }]\end{array}$} \\
\hline & $1^{\text {st }}$ pinion & $2^{\text {nd }}$ pinion & $3^{\text {rd }}$ pinion & $4^{\text {th }}$ pinion & Constant pinion & Rear pinion \\
\hline Module m & 3.4442 & 3.3707 & 3.2281 & 2.8278 & 3.6178 & 2.7138 \\
\hline Number of teeth $\mathrm{z}$ & 14.000 & 19.000 & 19.000 & 19.000 & 19.000 & 19.000 \\
\hline Helix angle $\beta$ & 32.000 & 30.7541 & 30.7453 & 30.7209 & 307695 & 31.7026 \\
\hline Face width $b$ & 34.000 & 33.000 & 32.000 & 32.000 & 32.000 & 44.000 \\
\hline Pressure angle $\alpha_{t}$ & 18.6816 & 18.4523 & 18.4507 & 18.4463 & 18.4550 & 18.6256 \\
\hline Centre distance a & 80.000 & 80.000 & 80.000 & 80.000 & 80.000 & 80.000 \\
\hline Transverse contact ratio $\varepsilon_{\alpha}$ & 1.5855 & 1.6184 & 1.6296 & 1.6603 & 1.5986 & 1.6589 \\
\hline Overlap ratio $\varepsilon_{\beta}$ & 1.6651 & & & & & \\
\hline Bending stress $\sigma_{\mathrm{F}}$ & 894.2161 & 738.8489 & 754.3754 & 610.8693 & 341.5908 & 928.0272 \\
\hline Safety factor for bending stress $S_{F}$ & 1.1183 & 1.3535 & 1.3256 & 1.6370 & 2.9275 & 1.0776 \\
\hline Contact stress $\sigma_{\mathrm{H}}$ & 977.500 & 828.500 & 772.000 & 743.700 & 582.200 & 1218.400 \\
\hline Safety factor for contact stress $\sigma_{\mathrm{H}}$ & 1.4323 & 1.6897 & 1.8134 & 1.8824 & 2.4046 & 1.1491 \\
\hline
\end{tabular}

(d)

Solution no 4 (pressure angle $\alpha=18^{\circ}$ )

Lower bound $\mathrm{Lb}=$ [as same as above]

Upper bound $\mathrm{Ub}=$ [as same as above]

Initial condition $\mathrm{X} 0=$ [as same as above $]$

\begin{tabular}{|c|c|c|c|c|c|c|}
\hline & $1^{\text {st }}$ pinion & $2^{\text {nd }}$ pinion & $3^{\text {rd }}$ pinion & $4^{\text {th }}$ pinion & Constant pinion & Rear pinion \\
\hline Module m & 3.4442 & 3.3703 & 3.2278 & 2.8275 & 3.6175 & 2.7137 \\
\hline Number of teeth $\mathrm{z}$ & 14.000 & 19.000 & 19.000 & 19.000 & 19.000 & 19.000 \\
\hline Helix angle $\beta$ & 32.000 & 30.7565 & 30.7478 & 30.7240 & 30.7716 & 31.7060 \\
\hline Face width $b$ & 34.000 & 33.000 & 32.000 & 32.000 & 32.000 & 44.000 \\
\hline Pressure angle $\alpha_{t}$ & 20.9637 & 20.7116 & 20.7099 & 20.7052 & 20.7146 & 20.9028 \\
\hline Centre distance a & 80.000 & 79.9942 & 79.9938 & 79.9922 & 79.9950 & 79.9996 \\
\hline Transverse contact ratio $\varepsilon_{\alpha}$ & 1.5077 & 1.5367 & 1.5459 & 1.5709 & 1.5202 & 1.5688 \\
\hline Overlap ratio $\varepsilon_{\beta}$ & 1.6651 & & & & & \\
\hline Bending stress $\sigma_{\mathrm{F}}$ & 927.6637 & 766.9720 & 783.5646 & 635.5152 & 354.2463 & 965.5743 \\
\hline Safety factor for bending stress $S_{F}$ & 1.0780 & 1.3038 & 1.2762 & 1.5735 & 2.8229 & 1.0357 \\
\hline Contact stress $\sigma_{\mathrm{H}}$ & 1000.240 & 850.300 & 792.700 & 764.600 & 597.100 & 1252.90 \\
\hline Safety factor for contact stress $\sigma_{\mathrm{H}}$ & 1.3967 & 1.6464 & 1.7661 & 1.8310 & 2.3448 & 1.1175 \\
\hline
\end{tabular}


(e)

\begin{tabular}{|c|c|c|c|c|c|c|}
\hline \multicolumn{7}{|c|}{$\begin{array}{c}\text { Solution no } 5 \text { (pressure angle } a=20^{\circ} \text { ) } \\
\text { Lower bound } \mathrm{Lb}=[\text { as same as above }] \\
\text { Upper bound } \mathrm{Ub}=[\text { as same as above }] \\
\text { Initial condition } \mathrm{X} 0=[\text { as same as above }]\end{array}$} \\
\hline & $1^{\text {st }}$ pinion & $2^{\text {nd }}$ pinion & $3^{\text {rd }}$ pinion & $4^{\text {th }}$ pinion & Constant pinion & Rear pinion \\
\hline Module m & 3.4442 & 3.3699 & 3.2274 & 2.8270 & 3.6172 & 2.7136 \\
\hline Number of teeth $\mathrm{z}$ & 14.000 & 19.000 & 19.000 & 19.000 & 19.000 & 19.000 \\
\hline Helix angle $\beta$ & 32.000 & 30.7584 & 30.7499 & 30.7265 & 30.7733 & 31.7088 \\
\hline Face width $b$ & 34.000 & 33.000 & 32.000 & 32.000 & 32.000 & 44.000 \\
\hline Pressure angle $\alpha_{t}$ & 23.2283 & 22.9551 & 22.9533 & 22.9483 & 22.9583 & 23.1628 \\
\hline Transverse contact ratio $\varepsilon_{\alpha}$ & 1.4417 & 1.4672 & 1.4749 & 1.4955 & 1.4535 & 1.4930 \\
\hline Overlap ratio $\varepsilon_{\beta}$ & 1.6651 & & & & & \\
\hline Bending stress $\sigma_{\mathrm{F}}$ & 958.800 & 793.400 & 811.000 & 658.700 & 366.100 & 1000.700 \\
\hline Safety factor for bending stress $\mathrm{S}_{\mathrm{F}}$ & 1.0429 & 1.2604 & 1.2331 & 1.5182 & 2.7314 & 0.9993 \\
\hline Contact stress $\sigma_{\mathrm{H}}$ & 1025.100 & 870.300 & 811.600 & 783.800 & 610.700 & 1284.300 \\
\hline Safety factor for contact stress $\sigma_{\mathrm{H}}$ & 1.3658 & 1.6087 & 1.7249 & 1.7862 & 2.2926 & 1.0901 \\
\hline
\end{tabular}

(f)

Solution no 6 (pressure angle $\alpha=22^{\circ}$ )

Lower bound $\mathrm{Lb}=$ [as same as above]

Upper bound $\mathrm{Ub}=$ [as same as above]

Initial condition $\mathrm{X} 0=[$ as same as above $]$

\begin{tabular}{|c|c|c|c|c|c|c|}
\hline & $1^{\text {st }}$ pinion & $2^{\text {nd }}$ pinion & $3^{\text {rd }}$ pinion & $4^{\text {th }}$ pinion & Constant pinion & Rear pinion \\
\hline Module m & 3.4442 & 3.3696 & 3.2271 & 2.8267 & 3.6169 & 2.7136 \\
\hline Number of teeth $z$ & 14.000 & 19.000 & 19.000 & 19.000 & 19.000 & 19.000 \\
\hline Helix angle $\beta$ & 32.000 & 30.7598 & 30.7514 & 30.7284 & 30.7745 & 31.7109 \\
\hline Face width $b$ & 34.000 & 33.000 & 32.000 & 32.000 & 32.000 & 44.000 \\
\hline Pressure angle $\alpha_{t}$ & 25.4740 & 25.1815 & 25.1796 & 25.1743 & 25.1849 & 25.4043 \\
\hline Centre distance a & 80.000 & 79.9806 & 79.9790 & 79.9735 & 79.9831 & 79.9988 \\
\hline Transverse contact ratio $\varepsilon_{\alpha}$ & 1.3862 & 1.4086 & 1.4150 & 1.4321 & 1.3970 & 1.4295 \\
\hline Overlap ratio $\varepsilon_{\beta}$ & 1.6651 & & & & & \\
\hline Bending stress $\sigma_{\mathrm{F}}$ & 987.300 & 817.700 & 836.200 & 680.000 & 377.000 & 1033.100 \\
\hline Safety factor for bending stress $S_{F}$ & 1.0128 & 1.2230 & 1.1958 & 1.4706 & 2.6522 & 0.9680 \\
\hline Contact stress $\sigma_{\mathrm{H}}$ & 1045.400 & 888.300 & 828.700 & 801.000 & 622.900 & 1312.500 \\
\hline Safety factor for contact stress $\sigma_{\mathrm{H}}$ & 1.3392 & 1.5761 & 1.6894 & 1.7478 & 2.2475 & 1.0667 \\
\hline
\end{tabular}

The results from solution 6 (Table 3) indicate that the obtained optimum values satisfy all requirements. However, by considering safety factors, this solution is more acceptable for $4^{\text {th }}$ speed. The safety factor for bending stress, $S_{F}$, ranges between 0.9680 and 2.6522 , and the safety factor for contact stress, $S_{H}$, varies between 1.0667 and 2.2475 . 
From the obtained optimisation results, it can be concluded that increasing the contact ratio results in reduced tooth bending stress and reduced contact stress. Furthermore, increased the pressure angle caused increased the tooth bending stress and contact stress, by reducing the contact ratio. The relations between the contact ratio and bending stress are shown in Figures 8-13. The contact ratio and pressure angle relations are shown in Figures 14-19.

\subsection{Contact Ratio and Tooth Bending Stress Relation}

The contact ratio and bending stress relation for the $1^{\text {st }}$ speed is shown in Figure 8. As the contact ratio increases from 1.3862 to 1.7836 , the bending stress reduces from $987.300\left[\mathrm{~N} / \mathrm{mm}^{2}\right]$ to $822.2394\left[\mathrm{~N} / \mathrm{mm}^{2}\right]$. Thus, increasing the contact ratio $28.66 \%$ results in a $20.07 \%$ reduction in tooth bending stress.

The contact ratio and bending stress relation for the $2^{\text {nd }}$ speed is shown in Figure 9. As the contact ratio for the $2^{\text {nd }}$ speed increases from 1.4086 to 1.8255 , the bending stress reduces from $817.7000\left[\mathrm{~N} / \mathrm{mm}^{2}\right]$ to $679.0009\left[\mathrm{~N} / \mathrm{mm}^{2}\right]$. Thus, increasing the contact ratio $29.59 \%$ reduces the tooth bending stress $20.42 \%$.

The contact ratio and bending stress relation for the $3^{\text {rd }}$ speed is shown in Figure 10. As the contact ratio for the $3^{\text {rd }}$ speed increases from 1.4150 to 1.8423 , bending stress reduces from $836.2000\left[\mathrm{~N} / \mathrm{mm}^{2}\right]$ to $692.3186\left[\mathrm{~N} / \mathrm{mm}^{2}\right]$. Thus, increasing the contact ratio $30.19 \%$, results a $20.78 \%$ reduction in tooth bending stress.

The contact ratio and bending stress relation for the $4^{\text {th }}$ speed is shown in Figure 11. As the contact ratio for the $4^{\text {th }}$ speed increases from 1.4321 to 1.8895 , the bending stress reduces from $680.0000\left[\mathrm{~N} / \mathrm{mm}^{2}\right]$ to $558.4413\left[\mathrm{~N} / \mathrm{mm}^{2}\right]$. Thus, increasing the contact ratio $31.93 \%$ reduces the tooth bending stress $21.76 \%$.

The contact ratio and bending stress relation for the $5^{\text {th }}$ speed is shown in Figure 12. As the contact ratio for the $5^{\text {th }}$ speed increases from 1.3970 to 1.7963 , the bending stress reduces from $377.0000\left[\mathrm{~N} / \mathrm{mm}^{2}\right]$ to $314.6310\left[\mathrm{~N} / \mathrm{mm}^{2}\right]$. Thus, increasing the contact ratio $28.58 \%$, results in a $19.82 \%$ reduction in the tooth bending stress.

The contact ratio and bending stress relation for the rear speed is shown in Figure 13. As the contact ratio for the rear speed increases from 1.4295 to 1.8913 , the bending stress reduces from $1033.1000\left[\mathrm{~N} / \mathrm{mm}^{2}\right]$ to 847.6631 $\left[\mathrm{N} / \mathrm{mm}^{2}\right]$. Thus, increasing the contact ratio $32.30 \%$, reduces the tooth bending stress $21.87 \%$.

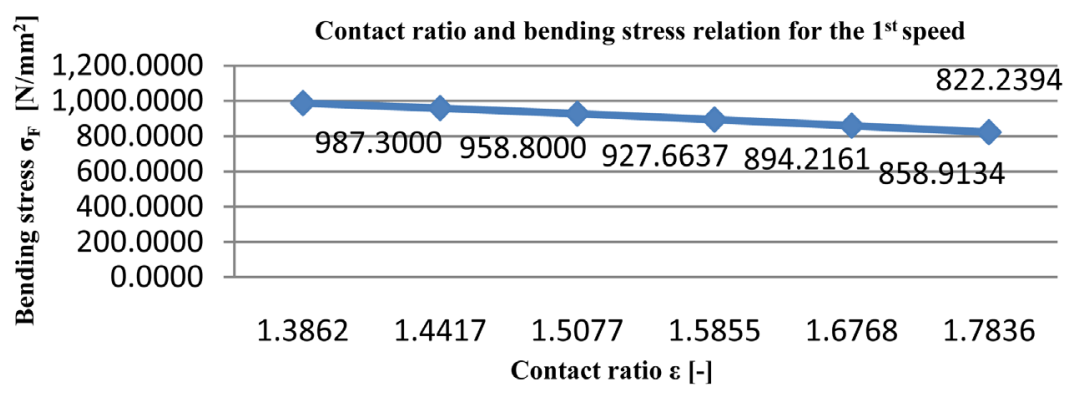

Figure 8. Contact ratio and bending stress relation for the $1^{\text {st }}$ speed. 


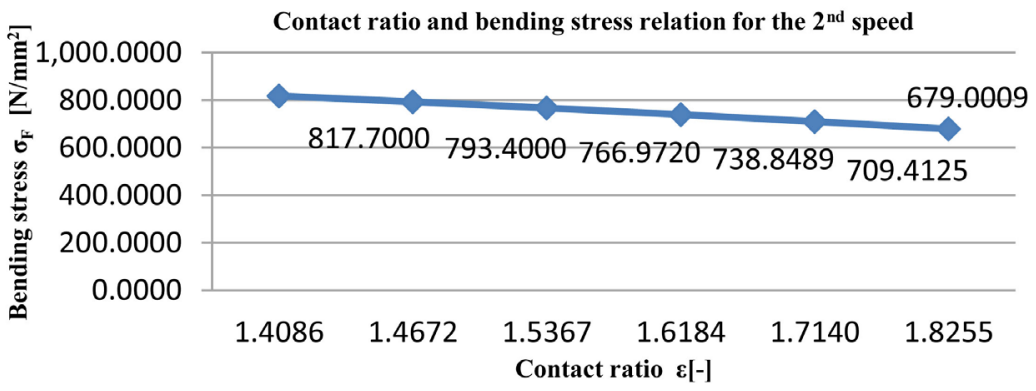

Figure 9. Contact ratio and bending stress relation for the $2^{\text {nd }}$ speed.

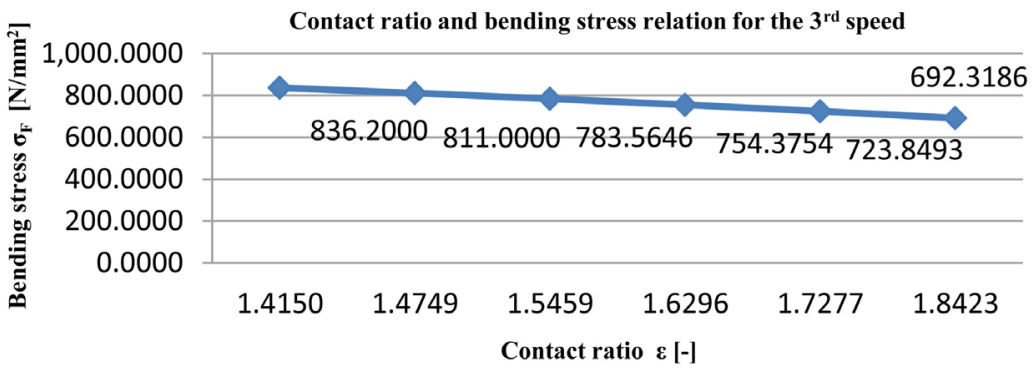

Figure 10. Contact ratio and bending stress relation for the $3^{\text {rd }}$ speed.

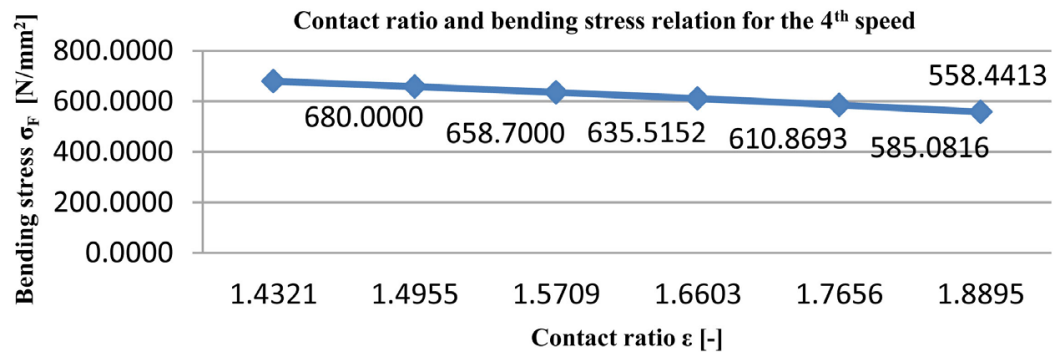

Figure 11. Contact ratio and bending stress relation for the $4^{\text {th }}$ speed.

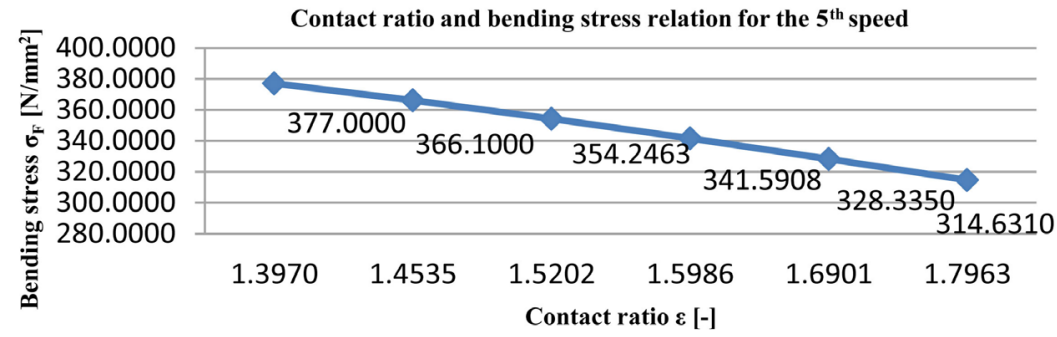

Figure 12. Contact ratio and bending stress relation for the $5^{\text {th }}$ speed.

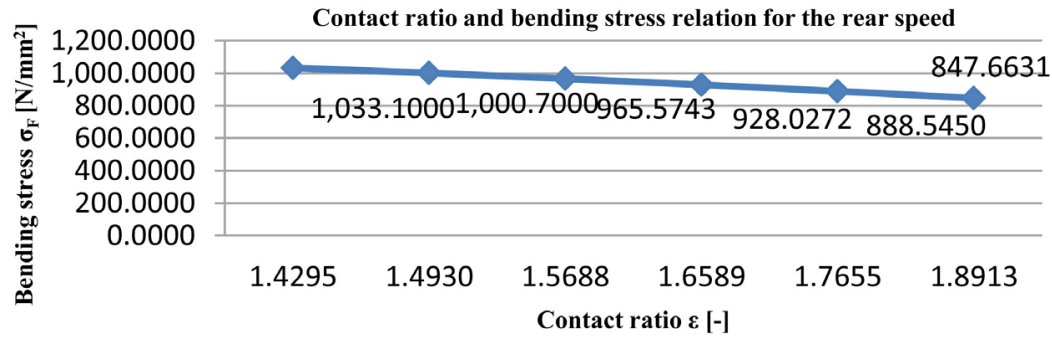

Figure 13. Contact ratio and bending stress relation for the rear speed. 


\subsection{Contact Ratio and Pressure Angle Relation}

The contact ratio and pressure angle relation for the $1^{\text {st }}$ speed is shown in Figure 14. As the pressure angle for the $1^{\text {st }}$ speed reduces from $22\left[^{\circ}\right]$ to $12\left[^{\circ}\right]$, the contact ratio increases from 1.3862 to 1.7836 . Thus, decreasing the pressure angle $83 \%$, results in a $28.66 \%$ increase in the contact ratio.

The contact ratio and pressure angle relation for the $2^{\text {nd }}$ speed is shown in Figure 15. As the pressure angle for the $2^{\text {nd }}$ speed reduces from $22\left[^{\circ}\right]$ to $12\left[^{\circ}\right]$, the contact ratio increases from 1.4086 to 1.8255 . Thus, decreasing the pressure angle $83 \%$, increases the contact ratio $29.59 \%$.

The contact ratio and pressure angle relation for the $3^{\text {rd }}$ speed is shown in Figure 16 . As the pressure angle for the $3^{\text {rd }}$ speed reduces from $22\left[{ }^{\circ}\right]$ to $12\left[{ }^{\circ}\right]$, the contact ratio increases from 1.4150 to 1.8423 . Thus, decreasing the pressure angle $83 \%$, results in a $30.19 \%$ increase in the contact ratio.

The contact ratio and pressure angle relation for the $4^{\text {th }}$ speed is shown in Figure 17. As the pressure angle for the $4^{\text {th }}$ speed reduces from $22\left[^{\circ}\right]$ to $12\left[^{\circ}\right]$, the contact ratio increases from 1.4321 to 1.8895 . Thus, decreasing the pressure angle $83 \%$, result in increases the contact ratio $31.93 \%$.

The contact ratio and pressure angle relation for the $5^{\text {th }}$ speed is shown in Figure 18. As the pressure angle for the $5^{\text {th }}$ speed reduces from $22\left[{ }^{\circ}\right]$ to $12\left[^{\circ}\right]$, the contact ratio increases from 1.3970 to 1.7963 . Thus, decreasing the pressure angle $83 \%$, results in a $28.58 \%$ increase in the contact ratio.

The contact ratio and pressure angle relation for the rear speed is shown in Figure 19. As the pressure angle for the rear speed reduces from $22\left[^{\circ}\right]$ to $12\left[^{\circ}\right]$, the contact ratio increases from 1.4295 to 1.8913 . Thus, decreasing the pressure angle $83 \%$, results in a $32.30 \%$ increase in the contact ratio.

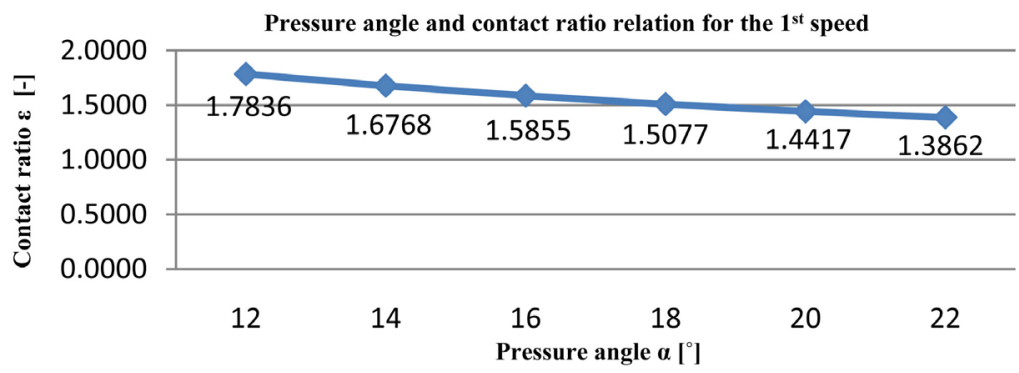

Figure 14. Contact ratio and pressure angle relation for the $1^{\text {st }}$ speed.

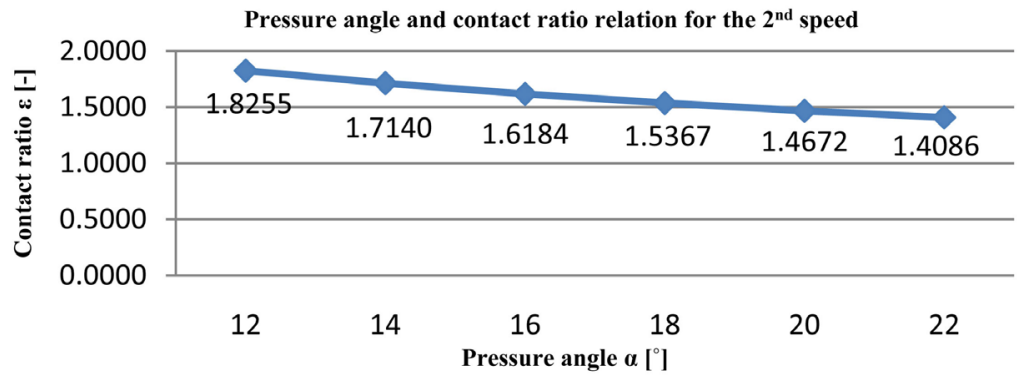

Figure 15. Contact ratio and pressure angle relation for the $2^{\text {nd }}$ speed. 


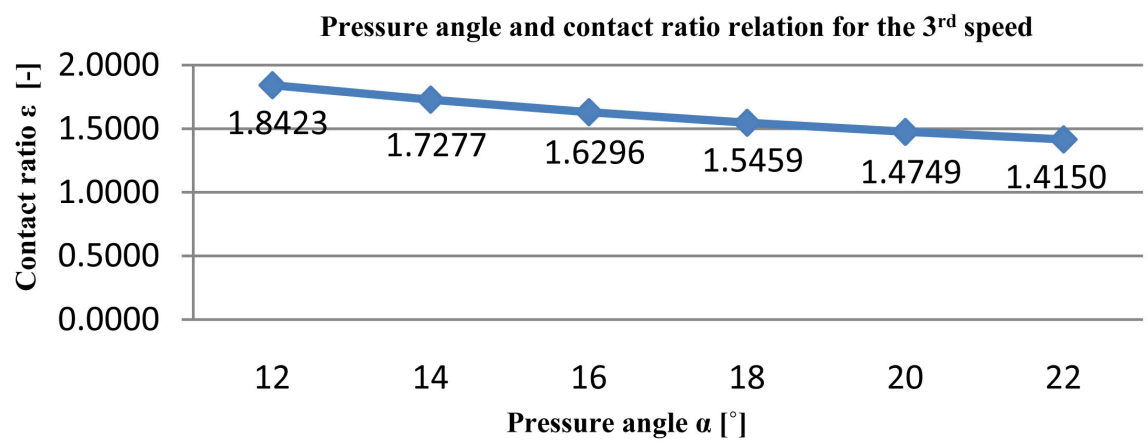

Figure 16. Contact ratio and pressure angle relation for the $3^{\text {rd }}$ speed.

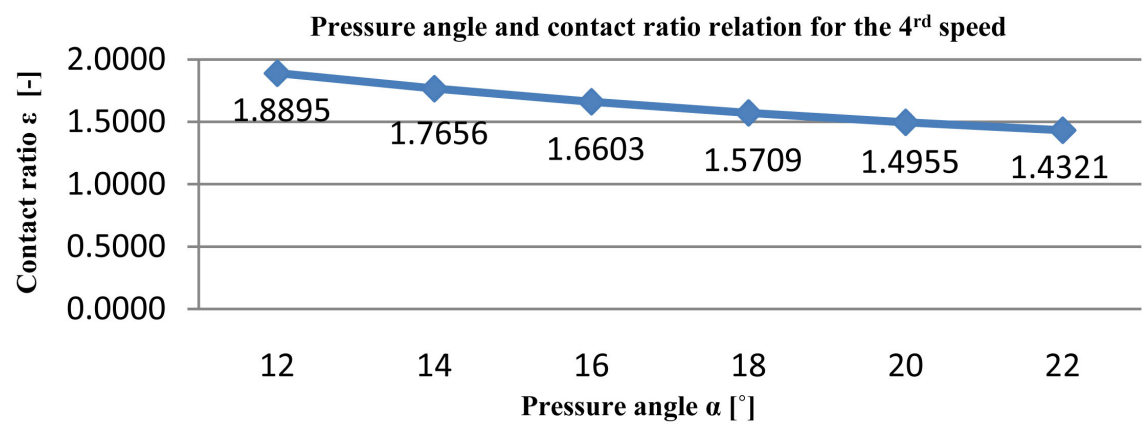

Figure 17. Contact ratio and pressure angle relation for the $4^{\text {th }}$ speed.

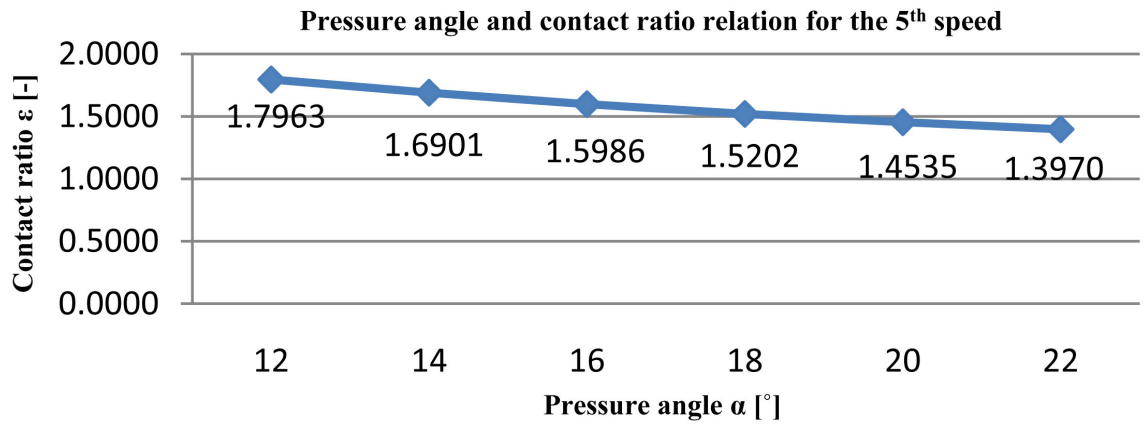

Figure 18. Contact ratio and pressure angle relation for the $5^{\text {th }}$ speed.

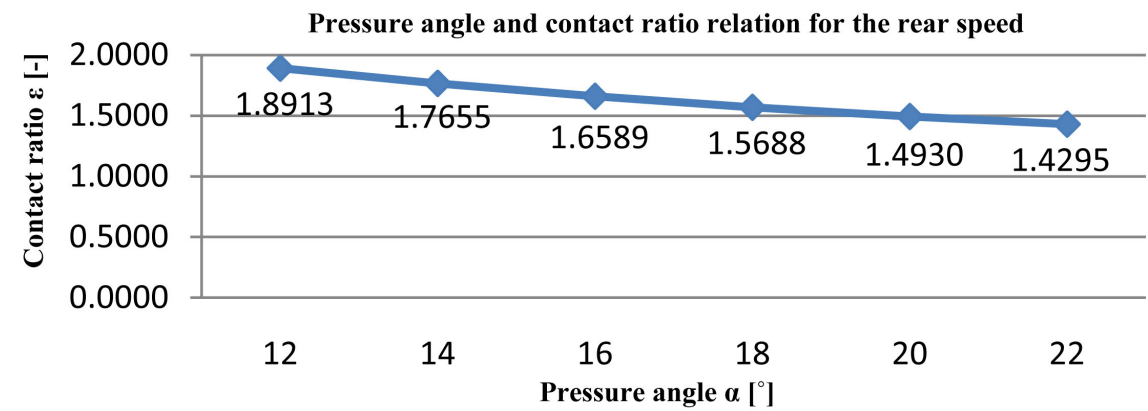

Figure 19. Contact ratio and pressure angle relation for the rear speed.

\subsection{Tooth Profile Modification Factor and Bending Stress Relation}

The tooth profile modification and bending stress relation for the $1^{\text {st }}$ speed is 
shown in Figure 20. While the profile modification factor increase from 0 to 0.3 , the bending stress reduces from $1171.5000\left[\mathrm{~N} / \mathrm{mm}^{2}\right]$ to $927.4486\left[\mathrm{~N} / \mathrm{mm}^{2}\right]$.

The tooth profile modification and bending stress relation for the $2^{\text {nd }}$ speed is shown in Figure 21. As the profile modification factor increase from 0 to 0.3 , the bending stress reduces from $854.7000\left[\mathrm{~N} / \mathrm{mm}^{2}\right]$ to $712.5610\left[\mathrm{~N} / \mathrm{mm}^{2}\right]$.

The tooth profile modification and bending stress relation for the $3^{\text {rd }}$ speed is shown in Figure 22. While the profile modification factor increase from 0 to 0.3 , the bending stress reduces from $873.9000\left[\mathrm{~N} / \mathrm{mm}^{2}\right]$ to $728.3622\left[\mathrm{~N} / \mathrm{mm}^{2}\right]$.

The tooth profile modification and bending stress relation for the $4^{\text {rd }}$ speed is shown in Figure 23. As the profile modification factor increase from 0 to 0.3 , the bending stress reduces from $709.5000\left[\mathrm{~N} / \mathrm{mm}^{2}\right]$ to $591.5892\left[\mathrm{~N} / \mathrm{mm}^{2}\right]$.

The tooth profile modification and bending stress relation for the $5^{\text {th }}$ speed is shown in Figure 24. As the profile modification factor increase from 0 to 0.3 , the bending stress reduces from $394.4000\left[\mathrm{~N} / \mathrm{mm}^{2}\right]$ to $328.8225\left[\mathrm{~N} / \mathrm{mm}^{2}\right]$.

The tooth profile modification and bending stress relation for the rear speed is shown in Figure 25. As the profile modification factor increase from 0 to 0.3 , the bending stress reduces from $1074.800\left[\mathrm{~N} / \mathrm{mm}^{2}\right]$ to $899.1084\left[\mathrm{~N} / \mathrm{mm}^{2}\right]$.

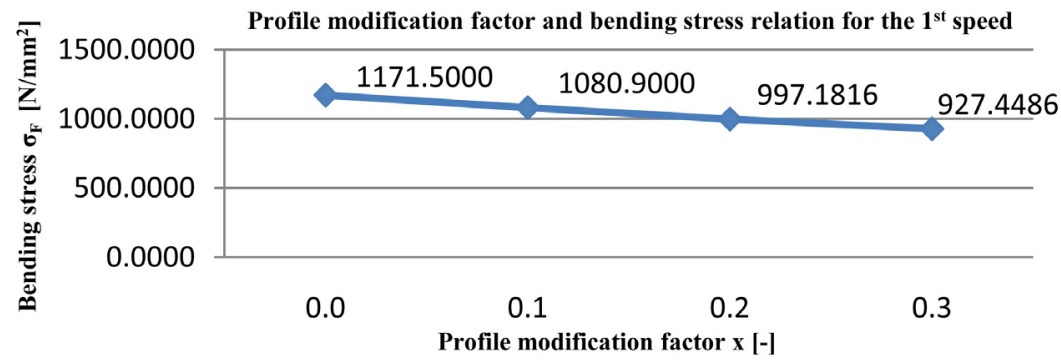

Figure 20. Profile modification factor and bending stress relation for the $1^{\text {st }}$ speed.

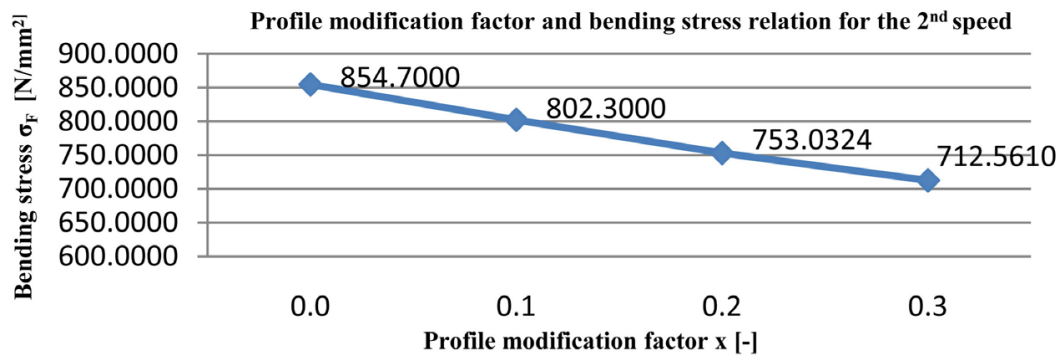

Figure 21. Profile modification factor and bending stress relation for the $2^{\text {nd }}$ speed.

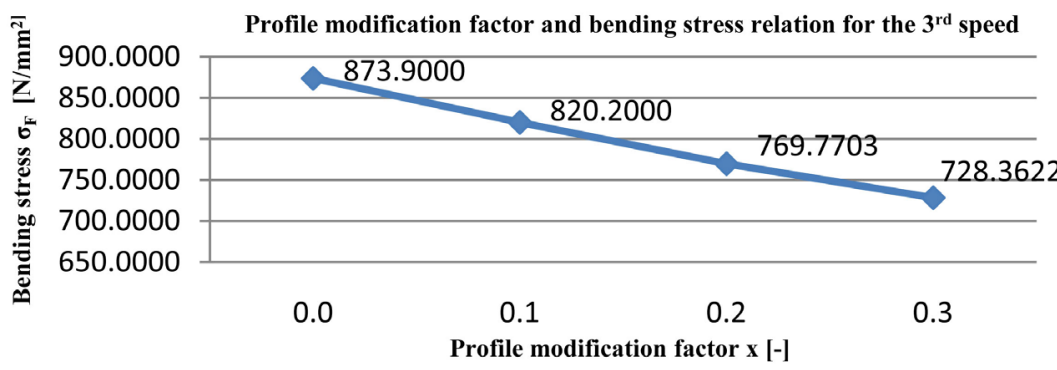

Figure 22. Profile modification factor and bending stress relation for the $3^{\text {rd }}$ speed. 


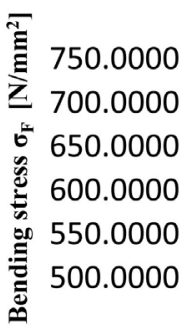

Profile modification factor and bending stress relation for the $4^{\text {rd }}$ speed

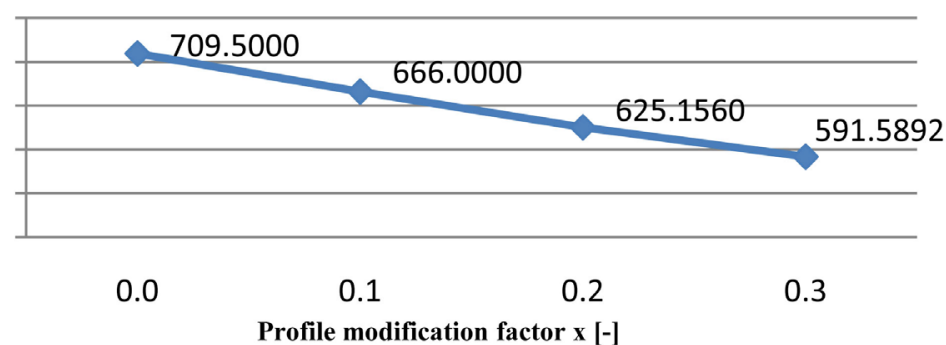

Figure 23. Profile modification factor and bending stress relation for the $4^{\text {th }}$ speed.

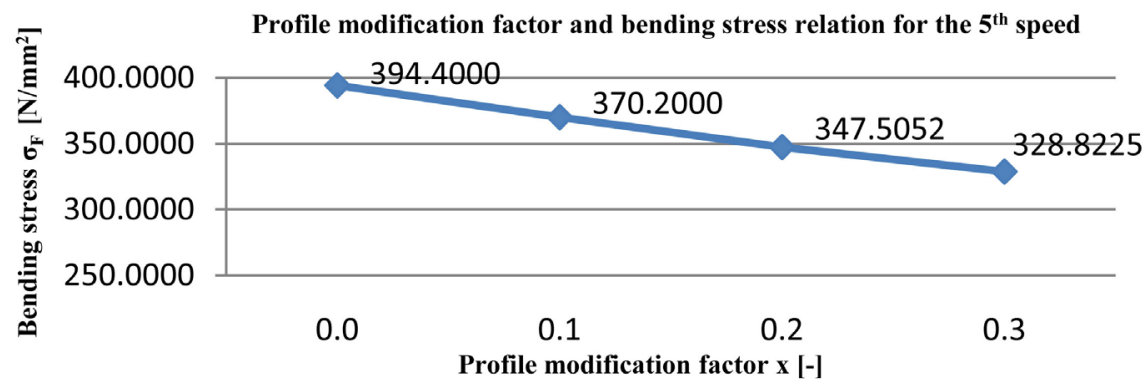

Figure 24. Profile modification factor and bending stress relation for the $5^{\text {th }}$ speed.

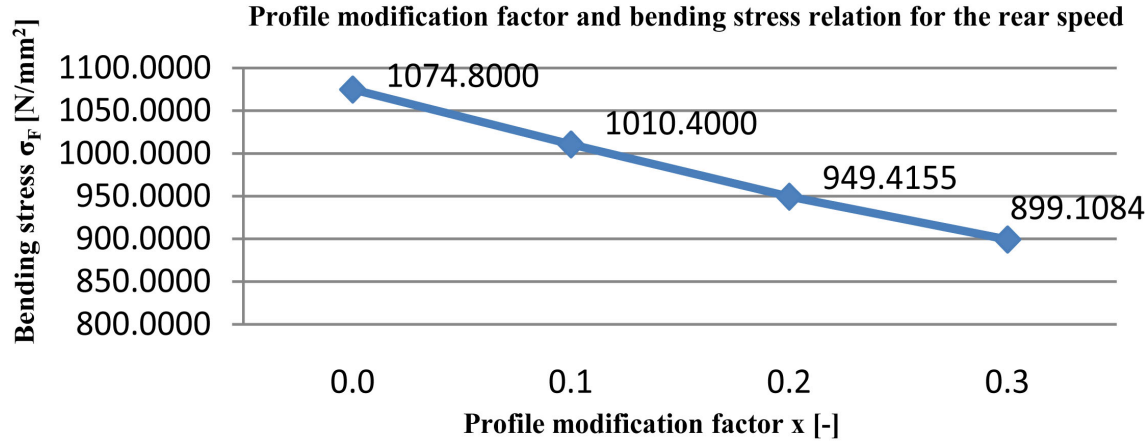

Figure 25. Profile modification factor and bending stress relation for the rear speed.

\subsection{Optimum Design of Effective Parameters}

A flowchart of the optimum design of effective parameters based on pressure angle is shown in Figure 26.

The safety factor for bending stress, $S_{F}$, and safety factor for contact stress $S_{H}$, are the basic selection criteria used by the Optimum Design. The Selective Optimum Design is shown in Table 4.

Although, obtained optimised geometric design parameters are significant for all constraints, the best solutions, based on pressure angle are determined from the obtained optimum solutions for each speed.

The geometric design parameters are optimised simultaneously for each given gearbox speed. However, it is not necessary to choose a single solution that changes with respect to the pressure angle. Therefore, all effective geometric design parameters can be determined independently for each speed from obtained optimum solutions. 


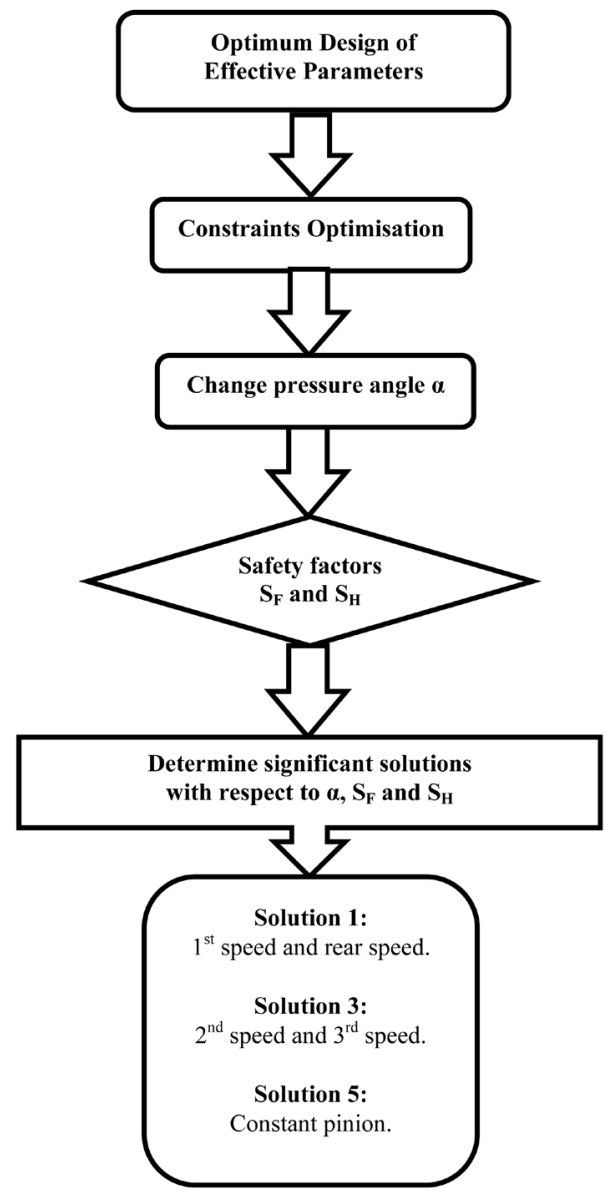

Figure 26. Flowchart of optimum design of effective parameters.

Table 4. Determination of best optimum solution.

\begin{tabular}{|c|c|c|c|c|c|c|}
\hline \multicolumn{7}{|c|}{ 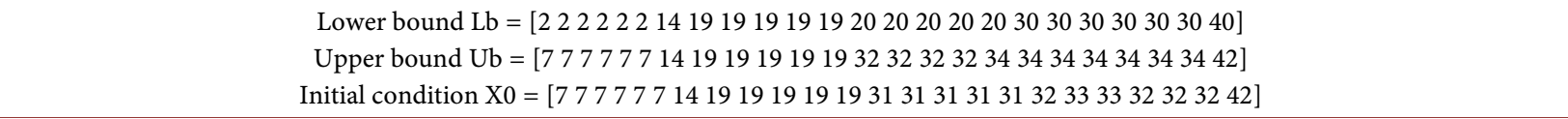 } \\
\hline & Sol.1 & Sol.3 & Sol.3 & Sol.3 & Sol.5 & Sol.1 \\
\hline & $\begin{array}{l}\text { Pressure angle } \\
\quad \alpha=12^{\circ}\end{array}$ & $\begin{array}{l}\text { Pressure angle } \\
\quad \alpha=16^{\circ}\end{array}$ & $\begin{array}{c}\text { Pressure angle } \\
\quad \alpha=16^{\circ}\end{array}$ & $\begin{array}{c}\text { Pressure angle } \\
\quad \alpha=22^{\circ}\end{array}$ & $\begin{array}{c}\text { Pressure angle } \\
\quad \alpha=20^{\circ}\end{array}$ & $\begin{array}{c}\text { Pressure angle } \\
\quad \alpha=12^{\circ}\end{array}$ \\
\hline & $1^{\text {st }}$ pinion & $2^{\text {nd }}$ pinion & $3^{\text {rd }}$ pinion & $4^{\text {th }}$ pinion & Constant pinion & Rear pinion \\
\hline Module m & 4.4442 & 3.3707 & 3.2281 & 2.8267 & 3.6172 & 2.7141 \\
\hline Number of teeth $\mathrm{z}$ & 14.000 & 19.000 & 19.000 & 19.000 & 19.000 & 19.000 \\
\hline Helix angle $\beta$ & 32.000 & 30.7541 & 30.7453 & 30.7284 & 30.7733 & 31.6943 \\
\hline Face width $b$ & 34.000 & 33.000 & 32.000 & 32.000 & 32.000 & 44.000 \\
\hline Pressure angle $\alpha_{t}$ & 14.0709 & 18.4523 & 18.4507 & 25.1743 & 22.9583 & 14.0261 \\
\hline Centre distance a & 80.000 & 80.000 & 80.000 & 79.9735 & 79.9885 & 80.000 \\
\hline Transverse contact ratio $\varepsilon_{\alpha}$ & 1.7836 & 1.6184 & 1.6296 & 1.4321 & 1.4535 & 1.8913 \\
\hline Overlap ratio $\varepsilon_{\beta}$ & 1.6651 & & & & & \\
\hline Bending stress $\sigma_{\mathrm{F}}$ & 822.2394 & 738.8489 & 754.3754 & 680.000 & 366.100 & 847.6631 \\
\hline Safety factor for bending stress $S_{F}$ & 1.2162 & 1.3535 & 1.3256 & 1.4706 & 2.7314 & 1.1797 \\
\hline Contact stress $\sigma_{\mathrm{H}}$ & 921.600 & 828.500 & 772.000 & 801.000 & 610.700 & 1141.100 \\
\hline Safety factor for contact stress $\sigma_{\mathrm{H}}$ & 1.5191 & 1.6897 & 1.8134 & 1.7478 & 2.2926 & 1.2269 \\
\hline
\end{tabular}




\section{Conclusions}

Optimisation of effective design parameters to reduce tooth bending stress for an automotive transmission gearbox is presented. The tooth bending stress is considered as the objective function, and the geometric design parameters are optimized under two different constraints. Tooth contact stress and constant distance between gear centres are considered as the constraints function. During optimization study, pressure angles were varied, thus contact ratios were also changed with respect to the pressure angle. The effect of the contact ratio on the tooth bending stress is analysed, and the following conclusions are drawn:

By optimising the effective geometric design parameters of the five-speed gearbox, such as the module, number of teeth, etc., reducing the tooth bending stress is possible.

Increasing the contact ratio results in reduced tooth bending stress and tooth contact stress. However, increased the pressure angle causes increasing of the tooth bending stress and tooth contact stress, since the contact ratio reduces depending on increasing of the pressure angle. Furthermore, higher contact ratio has a positive effect on reducing tooth bending stress. In contrast, higher pressure angle has a negative effect on reducing tooth bending stress. Application of tooth profile modification has a positive effectiveness on reducing the tooth bending stress.

Increasing the contact ratio $28.58 \%-32.30 \%$, results in a $19.82 \%-21.87 \%$ reduction in tooth bending stress. In contrast, decreasing the pressure angle $83 \%$, increases the contact ratio $28.58 \%-32.30 \%$. Gears with having higher contact ratio, have higher load carrying capacities.

Although, all the determined optimised geometric design parameters satisfy all constraints, it is not necessary to choose a single solution that changes with respect to the pressure angle.

All effective geometric design parameters can be determined independently for each speed inside the obtained optimum solutions. Based on pressure angle, the best optimised solutions are determined from the obtained optimum solutions for each speed in five-speed gearbox.

\section{References}

[1] Kumar, V.S., Muni, D.V. and Muthuveerappan, G. (2008) Optimization of Asymmetric Spur Gear Drives to Improve the Bending Load Capacity. Mechanism and Machine Theory, 43, 829-858.

[2] Costopoulos, T. and Spitas, V. (2009) Reduction of Gear Filet Stresses by Using One-Sided Involute Asymmetric Teeth. Mechanism and Machine Theory, 44, 15241534.

[3] Marimuthu, P. and Muthuveerappan, G. (2016) Design of Asymmetric Normal Contact Ratio Spur Gear Drive through Direct Design to Enhance the Load Carrying Capacity. Mechanism and Machine Theory, 95, 22-34.

[4] Pedrero, J.I., Pleguezuelos, M. and Munoz, M. (2011) Contact Stress Calculation of Undercut Spur and Helical Gear Teeth. Mechanism and Machine Theory, 46, 1633 1646. 
[5] Bozca, M. and Dikmen, F. (2012) Optimisation of Geometric Parameters of Gears under Variable Loading Condition. Advanced Materials Research, 445, 1005-1010. https://doi.org/10.4028/www.scientific.net/AMR.445.1005

[6] Juvinall, R.C. and Marshek, K.M. (2006) Fundamentals of Machine Component Design. John Wiley \& Sons, Inc., Hoboken.

[7] Naunheimer, H., Bertsche, B., Ryborz, J. and Novak, W. (2011) Automotive Transmissions. Springer-Verlag, Berlin Heidelberg. https://doi.org/10.1007/978-3-642-16214-5

[8] Roloff/Matek (2005) Maschinenelemente, Vieweg and Sohn Verlag. GWV Fachverlage $\mathrm{GmbH}$, Wiesbaden.

[9] Bozca, M. (2010) Torsional Vibration Model Based Optimization of Gearbox Geometric Design Parameters to Reduce Rattle Noise in an Automotive Transmission. Mechanism and Machine Theory, 45, 1583-1598.

[10] Bozca, M. and Fietkau, P. (2010) Empirical Model Based Optimization of Gearbox Geometric Design Parameters to Reduce Rattle Nose in an Automotive Transmission. Mechanism and Machine Theory, 45, 1599-1612.

Submit or recommend next manuscript to SCIRP and we will provide best service for you:

Accepting pre-submission inquiries through Email, Facebook, LinkedIn, Twitter, etc. A wide selection of journals (inclusive of 9 subjects, more than 200 journals)

Providing 24-hour high-quality service

User-friendly online submission system

Fair and swift peer-review system

Efficient typesetting and proofreading procedure

Display of the result of downloads and visits, as well as the number of cited articles

Maximum dissemination of your research work

Submit your manuscript at: http://papersubmission.scirp.org/

Or contact mme@scirp.org 